\title{
Pd grating obtained by direct micromolding for use in high resolution optical diffraction based sensing
}

\author{
RITU GUPTA and GIRIDHAR U KULKARNI* \\ Chemistry and Physics of Materials Unit, DST Unit on Nanoscience, Jawaharlal Nehru Centre for Advanced Scientific \\ Research, Jakkur P.O., Bangalore 560 064, India
}

MS received 14 December 2011; revised 28 April 2012

\begin{abstract}
Pd grating patterns have been fabricated using the process of micromolding in capillary employing a Pd alkanethiolate precursor, which could be converted to metal in situ by thermolysis. Thus generated Pd grating were uniform in width $(\sim 950 \mathrm{~nm})$ and spacing $(\sim 450 \mathrm{~nm})$ over millimeter square areas on glass substrates. Importantly, the pattern when used as an optical grating produced a diffraction pattern with a high resolution ( $>2000)$; the intensities of widely separated (diffraction angle, $\sim 26 \cdot 8^{\circ}$ ) diffracted spots could be measured using a simple photodiode. By varying the concentration of Pd precursor ( $2 \mathrm{mM}$ to $25 \mathrm{mM}$ ), thickness of the resulting gratings could be adjusted in the range of $\sim 15-115 \mathrm{~nm}$. By adjusting the grating parameters optimally, a maximum diffraction efficiency of 36\% has been achieved. Thus fabricated Pd grating was used as seed catalyst to deposit Cu by electroless plating. The $\mathrm{Cu}$ deposition process has also been monitored by employing AFM, SEM and EDS analysis. The diffraction efficiency values corroborate well with the changes in the grating thickness due to Cu deposition. The grating structures presented can be reproducibly fabricated for rapidly emerging optical diffraction based sensing applications. This has been demonstrated in the case of aqueous $\mathrm{Cu}^{2+}$ by depositing the latter electrolessly on Pd.
\end{abstract}

Keywords. Micromolding technique; Pd grating; Cu electroless deposition; diffraction efficiency; optical diffraction.

\section{Introduction}

Optical diffraction based sensing has emerged as a simple yet promising detection tool. Minute changes in the diffraction intensity can be used as a sensor signal for events taking place on the grating surface (Ryckman et al 2010). For instance, living cells (Morhard et al 2000) and large biomolecules (Tsay et al 1991; St. John et al 1998; Goh et al 2002) have been detected with fairly good sensitivity employing few tens of micron sized diffraction gratings. The binding ability of various analytes with the molecules of interest has been accessed using in situ assembled diffraction gratings where thickness variation of the grating and changes in refractive index were studied (Goh et al 2005). In this context, Acharya et al (2007a, b, 2008) have carried out biomolecular studies of various diseases and bacterial cells. Bailey and Hupp (2003) and Bailey et al (2003) performed sensing of volatile compounds, DNA as well as electrochemically induced changes in polymers using chemoresponsive diffraction gratings where changes in refractive index was monitored (Massari et al 2001). The growing interest in this technique is evident from diverse types of grating surfaces that have been explored as sensor material. For example, Halpern et al (2009) reported fabrication of ordered arrays

\footnotetext{
*Author for correspondence (kulkarni@jncasr.ac.in)
}

of $\mathrm{Au}$ and $\mathrm{Ni}$ gratings on a transparent surface whereas Shallcross et al (2009) patterned CdSe nanocrystals using microcontact molding for the fabrication of transmission gratings. Above literature methods mostly differ in the grating structure, grating component material and geometry in which diffraction is studied. The diffractometry set up is simple in the transmission mode with only 3 basic requirements: a laser source, a sample grating and a detector. An inexpensive laser source of power as low as $1 \mathrm{~mW}$ should suffice for this purpose. The nature of grating is highly varied from simple grating with parallel set of lines with widths and spacings in the range of few microns are commonly used. The diffraction intensity not only depends on refractive index of the surrounding medium but also on the grating parameters such as the width, spacing and thickness of the diffractive optical element.

The grating equation is generally given by

$$
\sin \theta-\sin \gamma=m \frac{\lambda}{D}(m= \pm 1, \pm 2),
$$

where $D$ is the grating period $(\sim 1.5 \mu \mathrm{m}), m$ the order of interference, $\lambda$ the wavelength of light used, $\theta$ the incidence angle and $\gamma$ the diffraction angle. The diffraction efficiency $(\mathrm{DE}(\%))$ of the grating is calculated as the ratio of first order intensity to the zero order (Acharya et al 2007a, b; Bailey and Hupp 2003; Bailey et al 2003). The smaller the value of $D$, higher will be angular divergence of the diffraction thus enabling use of simple point photodetectors instead of $2 \mathrm{D}$ 
CCD camera or any other expensive instrumentation. There has been an upsurge of activity in this direction to create high resolution gratings with grating element close to the wavelength of light employed (Chen et al 2010). Similarly, there is impetus to fabricate gratings with optimized grating parameters for achieving higher diffraction efficiencies and sensitivities and also for miniaturization of the sensor device (Sarov et al 2007). The sophistication in grating structures thus calls for advanced lithography fabrication tools. Photolithography is routinely employed to make grating patterns (Sendroiu and Corn 2008). Conventional soft lithographic methods such as $\mu$-contact printing (Kane et al 1999), have been most commonly used to fabricate low cost diffraction gratings. Multiple beam interference (Konkola et al 2003) and electron beam lithography (Bhuvana and Kulkarni 2008) though have capability of patterning over large areas but are expensive techniques. The fabrication of optically transmitting diffraction grating is still challenging, major problems being high absorptivity and diffusivity along with poor adhesion of molecular inks to the transparent substrate (Aithal et al 2006; Inberg et al 2009). Transmission gratings made of free standing $\mathrm{ZnO}$ nanowires have been fabricated by Pan et al (2005) for applications as diffraction based beam splitters.

In this article, we report a simple way of fabricating a high resolution metal grating based on a direct micromolding in capillaries. Pd alkanethiolate is used as single precursor source for molding in the capillary channels of a PDMS mold. Thus created Pd grating has been demonstrated as an easy and low inexpensive optical sensing element for diffraction based detection. As an example, electroless deposition of $\mathrm{Cu}$ was carried out where the extent of deposition was estimated by measuring the $\mathrm{DE}$ of the modified grating. The utilization of grating produced by direct micromolding for this technique may find applications in electronic industry for variety of optoelectronics applications such as to monitor $\mathrm{Cu}$ deposition on Pd seed layer.

\section{Experimental}

\subsection{Fabrication of mold for patterning}

The process of fabrication of Pd grating is illustrated in figure 1a. Elastomeric stamps were fabricated by replica molding of polydimethylsiloxane (PDMS) on a commercially available compact disk (Sony CD-R). PDMS was prepared by mixing Sylgard-184 elastomer and the curing agent (Dow Corning) in the ratio 10:1. The mixture was sonicated at room temperature to reduce air bubbles and then degassed under vacuum for $1 \mathrm{~h}$. PDMS mixture was poured onto the master (CD) and then cured in an oven at $60^{\circ} \mathrm{C}$ for $6 \mathrm{~h}$. PDMS stamps were cleaned using hexane and sonicated in ethanol to remove any uncured polymer. The resulting stamp hosted relief features of width $500 \pm 5 \mathrm{~nm}$ with intermediate channels of $1000 \pm 5 \mathrm{~nm}$ width.

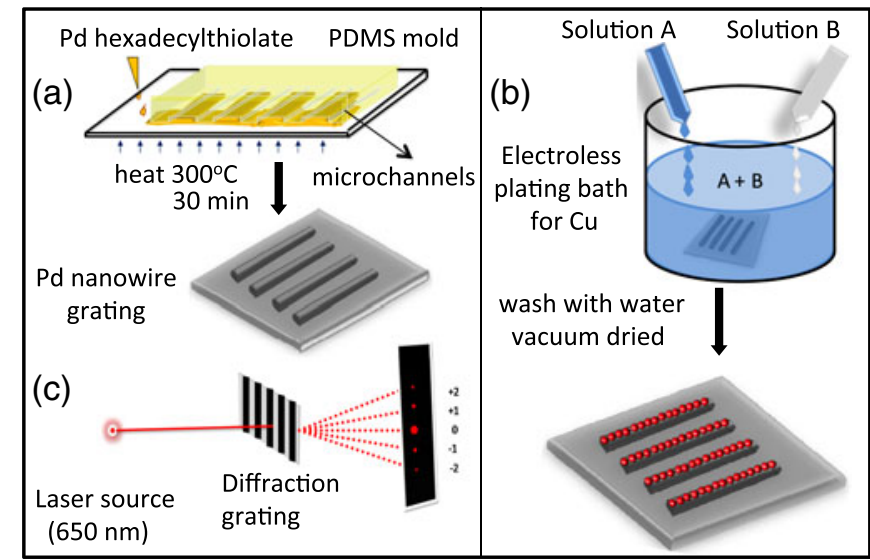

Figure 1. (a) Schematic illustration of procedure used for fabricating Pd grating, (b) electroless deposition of $\mathrm{Cu}$ on $\mathrm{Pd}$ grating and (c) diffraction set up.

\subsection{Preparation of 'ink'-Pd hexadecylthiolate}

The precursor employed for patterning was Pd hexadecylthiolate, $\mathrm{Pd}\left(\mathrm{SC}_{16} \mathrm{H}_{33}\right)_{2}$, in toluene. The synthesis of precursor involves a single step procedure. $\mathrm{Pd}(\mathrm{OAc})_{2}(5.0 \mathrm{mmol})$ in $7 \mathrm{ml}$ toluene was added to $5.0 \mathrm{mmol}$ of hexadecylthiol in toluene $(3 \mathrm{ml})$, and the resulting mixture was stirred vigorously overnight resulting in a yellow viscous colour solution which further deepened to orange-yellow. The obtained thiolate was washed with methanol followed by precipitation to remove excess thiol. The solid was redissolved to obtain a toluene solution of varied concentrations (2$25 \mathrm{mM}$ ). Pd hexadecylthiolate was chosen as 'ink' for micromolding because it readily dissolves in organic solvents such as toluene and remains stable under ambient for longer time periods. Under the molding conditions at $250^{\circ} \mathrm{C}$, the 'ink' undergoes thermolysis to give rise to Pd metal grating structures (Radha and Kulkarni 2009).

\subsection{Patterning of Pd grating pattern}

The glass slide used was cut into small pieces of $1 \times 1 \mathrm{~cm}^{2}$ and cleaned by sonicating in acetone and double-distilled water and dried under flowing argon. $30 \mu \mathrm{L}$ of the precursor solution (2-25 $\mathrm{mM}$ in toluene) was dropped at one edge of the PDMS stamp kept on the substrate to fill the channels spontaneously by capillary action as seen in figure 1a. The set up was gradually heated on a hot plate to reach $250^{\circ} \mathrm{C}$ and was held for $30 \mathrm{~min}$. Following cooling to room temperature, the stamp was removed leaving behind patterns on the substrate (figure 1a).

\subsection{Electroless deposition of $\mathrm{Cu}$ on $\mathrm{Pd}$ grating}

The deposition of $\mathrm{Cu}$ was initiated in an autocatalytic manner on the patterned Pd surface. The copper plating bath used for electroless $\mathrm{Cu}$ deposition consisted of solution A (1.5 g 
of $\mathrm{CuSO}_{4}, 7 \mathrm{~g}$ of $\mathrm{KNaC}_{4} \mathrm{H}_{4} \mathrm{O}_{6} \cdot 4 \mathrm{H}_{2} \mathrm{O}$ and $7 \mathrm{~g}$ of $\mathrm{NaOH}$ in $50 \mathrm{ml}$ water) and solution $\mathrm{B}(37.2 \mathrm{wt} \%$ aqueous formaldehyde solution) (Hidber et al 1996). The two solutions were taken in 10:1 (v/v) ratio and mixed together just before electroless plating was performed as shown in figure 1b. Each substrate was taken out after a predetermined time interval and immersed in a water bath to arrest the reaction. The substrates were vacuum dried prior to diffraction experiments. The electroless bath was freshly prepared in each case to avoid influence of aging.

\subsection{Optical diffraction studies}

A circular grating area slightly larger than the beam size $(0.5 \mathrm{~cm})$ was defined by scratching away rest of the patterned metal with a razor blade. The laser beam was aligned with respect to the designated circular area with the help of a monocular eyepiece. The diffraction experiments were performed using a simple optical set up as shown in figure 1c. The laser beam $(650 \mathrm{~nm}, 3 \mathrm{~mW})$ was directed normal to the grating surface and the transmitted light after diffraction was measured using a photodiode (Thorlabs, DET 200) connected to digital oscilloscope (Hewlett Packard-54600B, $100 \mathrm{MHz}$ ). The intensity of diffraction spots was measured from a fixed distance $(3 \mathrm{~cm})$ from the sample by placing the photodetector adjacent to screen position. In addition, diffraction images were photographed using a digital camera.

\subsection{Characterization of $\mathrm{Pd}$ and $\mathrm{Pd} / \mathrm{Cu}$ grating}

AFM measurements were performed using Veeco di Innova SPM in the tapping mode. Standard Si cantilevers were used for normal topography imaging. The surface roughness was estimated from AFM images using offline software, Nanoscope v7.30. The patterned substrates were also examined under a Nova NanoSEM 600 instrument (FEI Co., The Netherlands). Energy-dispersive spectroscopy (EDS) analysis was performed with an EDAX Genesis instrument (Mahwah, NJ) attached to the SEM column.

\section{Results and discussion}

The periodic line pattern seen in AFM images in figure $2 \mathrm{a}$ is the Pd grating from micromolding of Pd hexadecylthiolate. The wires are continuous, nearly smooth and uniform over several hundred micron square areas (see figure S1). From figure $2 \mathrm{a}$, we observe that the width of the wires is $\sim 450 \mathrm{~nm}$ and spacing, $\sim 1050 \mathrm{~nm}$ in close compliance with the PDMS stamp features. Line grating of different thicknesses could be obtained by micromolding with varying concentrations of the precursor solution viz. 2, 5, 10, 15, 20 and $25 \mathrm{mM}$, keeping other molding conditions constant (see $\S 2$ ). The surface morphology of the obtained patterns is shown in supplementary figures S2(a)-(f). The roughness of the grating structures was in the range of $\sim 15-25 \mathrm{~nm}$.

The thickness of the grating was found to increase with increasing concentration of the precursor solution, from $\sim 15 \mathrm{~nm}$ with $2 \mathrm{mM}$ solution to $\sim 115 \mathrm{~nm}$ with $25 \mathrm{mM}$. The variation is gradual and deviates slightly from being linear (figure 2b), thus giving us a good control. With the laser beam incident perpendicular to the plane of the grating lines (see figure 1c), optical diffraction patterns exhibiting well resolved diffraction spots were obtained in the transmission mode in each case. One such example is shown in the inset of figure $2 \mathrm{~b}$ for grating of $35 \mathrm{~nm}$ thickness. The resolution depends upon the illuminated area of the grating, the grating width, the wavelength of light and the incident angle (Nakajima et al 1999). For the above gratings, with the laser beam $(\lambda=650 \mathrm{~nm})$ incident normally over a grating area of $\sim 1 \mathrm{~mm}$ radius, the resolution works out to be $\sim 2000$. Such high resolution owes much to the narrow grating spacing $(450 \mathrm{~nm})$, a value which is much smaller compared to micron wide conventional gratings. The diffraction angle obtained experimentally for the first order diffraction spot is $\sim 26 \cdot 8^{\circ}$ (a)

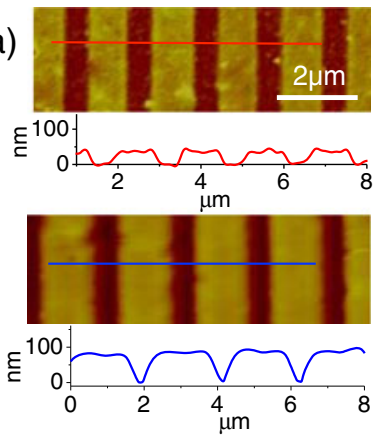

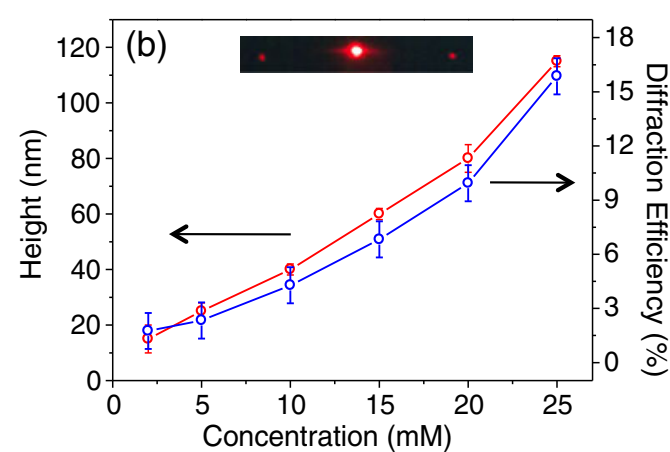

Figure 2. (a) AFM images of Pd gratings of thickness, $35 \mathrm{~nm}$ (top) and $90 \mathrm{~nm}$ (bottom) on glass substrates, along with thickness profiles and (b) variation in grating thickness and diffraction efficiency with precursor concentrations. Inset shows diffraction pattern from a grating with $35 \mathrm{~nm}$ thickness. 
which is slightly lower than the calculated angle $\left(27.7^{\circ}\right)$. Interestingly, the diffraction efficiency values for the different grating structures shown in figure $2 b$, vary in accordance with the grating thickness. Pd grating with a thickness of $82 \mathrm{~nm}$ was chosen for further measurements since the diffraction spots are visibly intense and more easily measurable. The initial diffraction efficiency was found to be $\sim 11 \%$ for this grating.

Figure 3 depicts the plot of diffraction efficiency as a function of $\mathrm{Cu}$ deposition time. The values of $I_{1}$ and $I_{0}$ used in DE calculation were the average values from 7 different areas of the sample. As shown in insets of figure 3, the diffraction patterns consist of sharp spots, the intensity diminishing at higher deposition times. Accordingly, the diffraction efficiency is seen to increase up to $120 \mathrm{~s}$ and falls off sharply thereafter, to reach near zero at $210 \mathrm{~s}$.

The associated changes in the grating structure were examined using SEM and EDS. From the SEM image shown in figure $4 \mathrm{c}, \mathrm{Cu}$ deposits on the Pd grating stripes can be clearly seen (compared with pristine grating in figure $4 a$ ). Interestingly, growth of $\mathrm{Cu}$ on $\mathrm{Pd}$ takes place only at the regions wherever Pd is present and this way, non-specific deposition is prevented. Based on $\mathrm{Cu} \mathrm{K}$ EDS signal (figure 4d) from 3 different areas, the atom percentage values were calculated. The amount of deposition is less in the beginning, which increases sharply after $120 \mathrm{~s}$ of dipping in electroless bath (figure $4 \mathrm{e}$ ). As shown in the scheme in the inset, the latter situation may correspond to $\mathrm{Cu}$ deposits around the Pd grating thickness.

The grating structures were further characterized using AFM (figure 5). In the initial stages (60 s, figure 5a), $\mathrm{Cu}$ deposits are seen nearly uniform over Pd grating surface. With increased time intervals, the topography became quite rough with $\mathrm{Cu}$ features projecting out from the surface (figures $5 \mathrm{~b}$ and $\mathrm{c}$ ). The average surface roughness

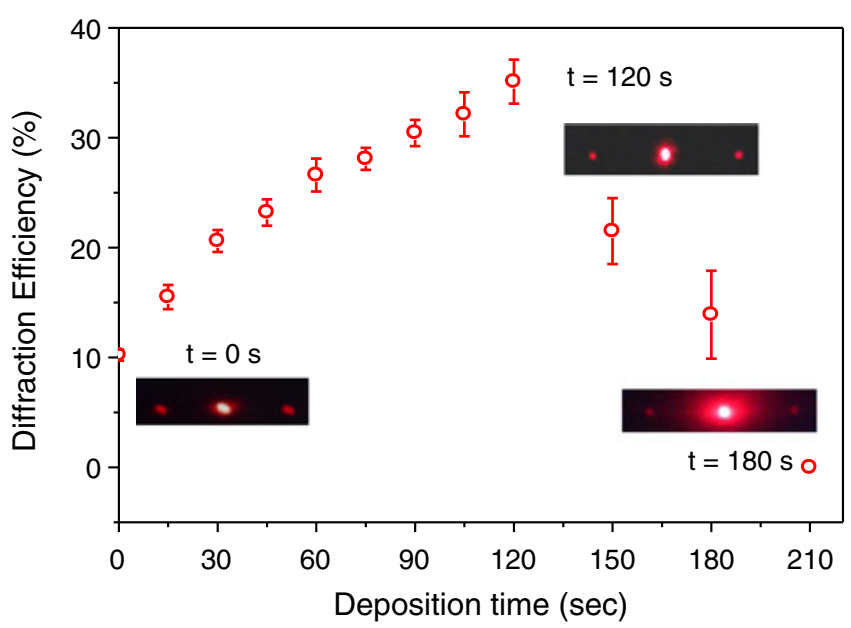

Figure 3. Variation in diffraction efficiency (DE\%) with plating time for $\mathrm{Cu}$ deposition. Some diffraction patterns are shown alongside.
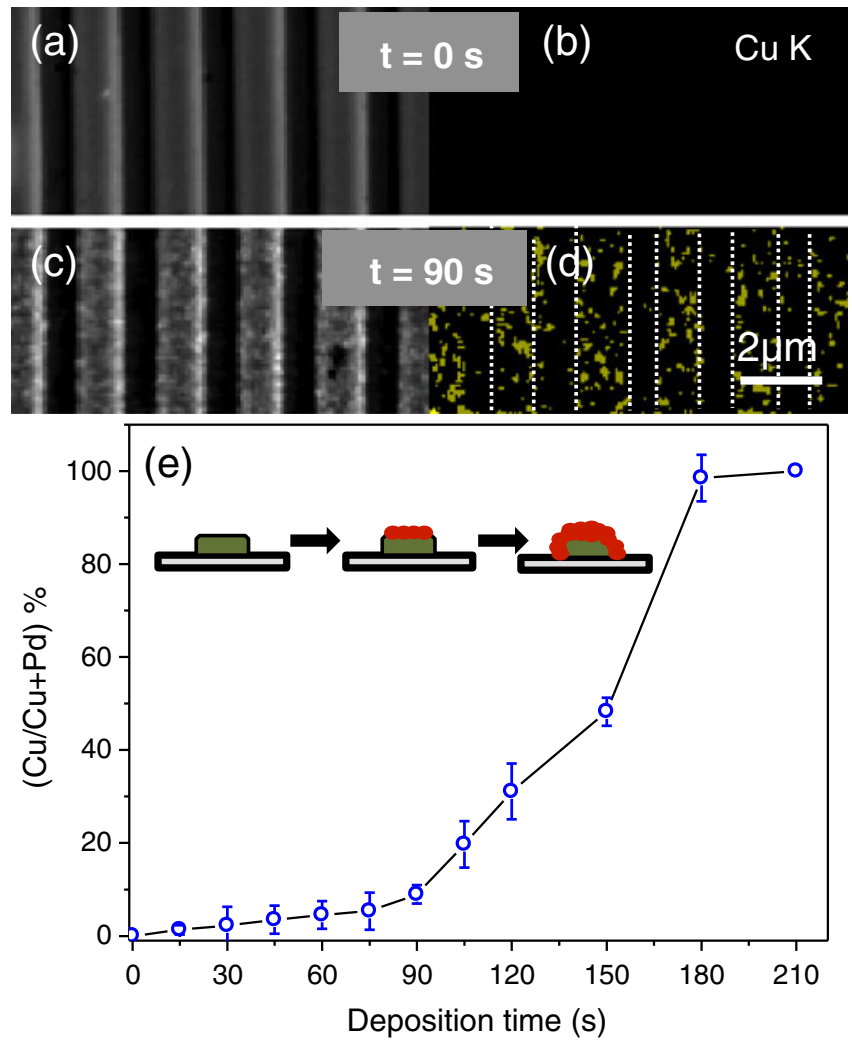

Figure 4. SEM micrographs (left) and corresponding Cu K EDS maps (right) before (a and b) and after dipping in $\mathrm{Cu}$ plating solution for $90 \mathrm{~s} \mathrm{(c} \mathrm{and} \mathrm{d)} \mathrm{and} \mathrm{(e)} \mathrm{atom} \mathrm{percentage} \mathrm{of} \mathrm{Cu}$ deposited over Pd with plating time.

determined from several $10 \times 10 \mu \mathrm{m}$ areas, increased from $14.5 \pm 2.9 \mathrm{~nm}$ to $60.3 \pm 3.4 \mathrm{~nm}$ after $120 \mathrm{~s}$ of Cu deposition. Correspondingly, the diffraction pattern became increasingly diffused (see figure 3, inset). Beyond $180 \mathrm{~s}$ however, there was a dramatic increase in grating surface roughness making further AFM characterization difficult (figure 5d). At this stage, there was no visible diffraction pattern (see figure 3 , inset). The thickness of grating increased from 78 to $90 \mathrm{~nm}$ after $60 \mathrm{~s}$, as $\mathrm{Cu}$ deposition was initiated, while the increase was much rapid at higher deposition time intervals (figure 5e). The reduction and deposition of $\mathrm{Cu}$ comes down after saturation point (180 s). Clearly, Cu deposition on Pd should be more efficient than $\mathrm{Cu}$ on $\mathrm{Cu}$. What is noteworthy is that the deposition already covers the active range (few nms to hundred nms) for most deposition processes.

The error values have been estimated from standard deviations in the thickness and width values from different areas of the samples. The $\mathrm{Cu}$ deposition had relatively small effect on the grating width (figure $5 \mathrm{f}$ ).

The thickness of grating as measured from AFM can be directly correlated to the amount of $\mathrm{Cu}$ deposited as can be seen in figure 6. It is observed that the thickness of grating increases proportionally with increasing amount of $\mathrm{Cu}$ deposition up to $180 \mathrm{~s}(\sim 50 \%)$. The intensity of light 

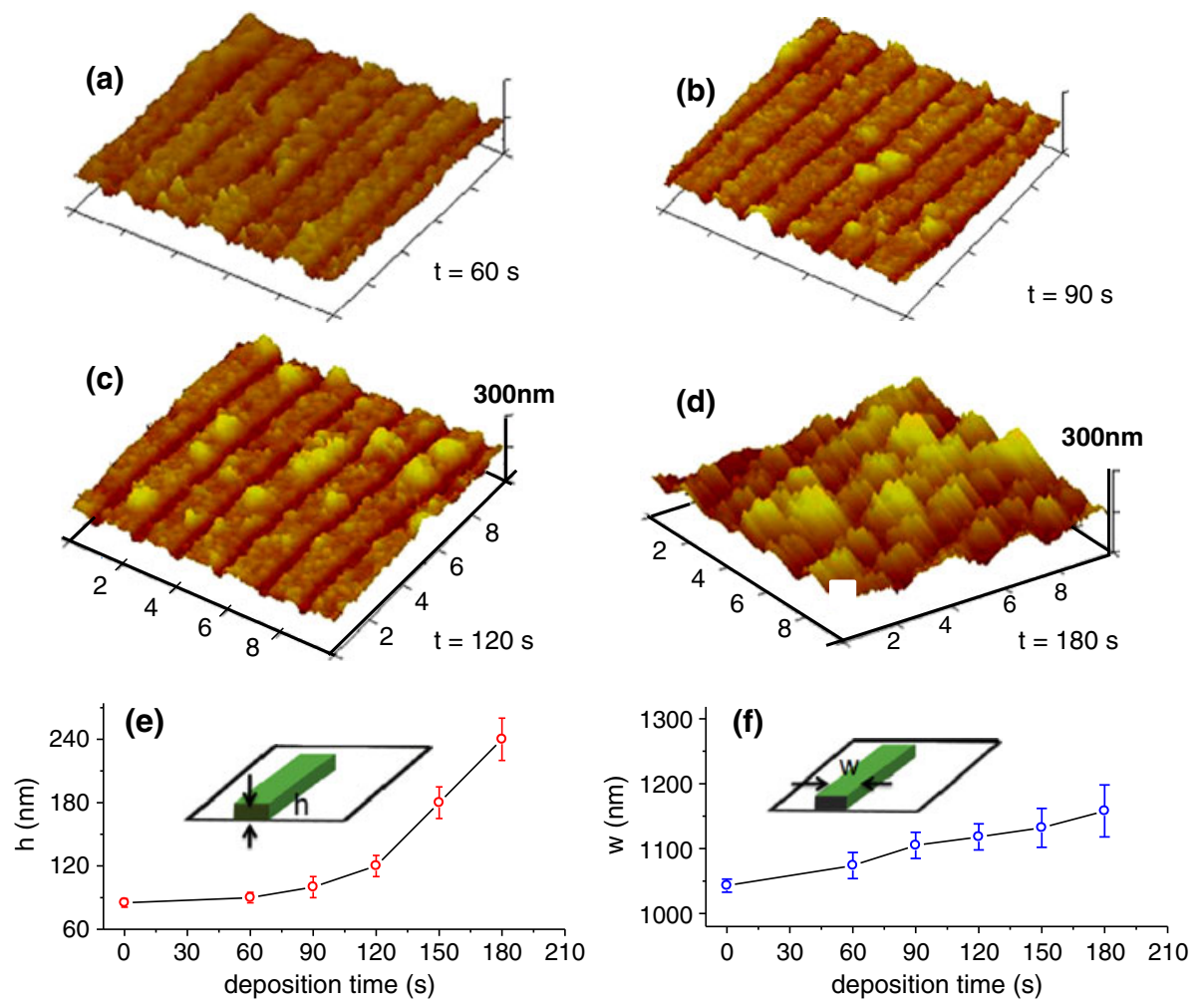

Figure 5. (a)-(d) AFM images of Pd gratings after dipping in Cu plating solution for varying time intervals of (a) $60 \mathrm{~s}$, (b) $90 \mathrm{~s}$, (c) $120 \mathrm{~s}$ and (d) $180 \mathrm{~s}$. Variations in (e) thickness and (f) width of grating with $\mathrm{Cu}$ deposition time.

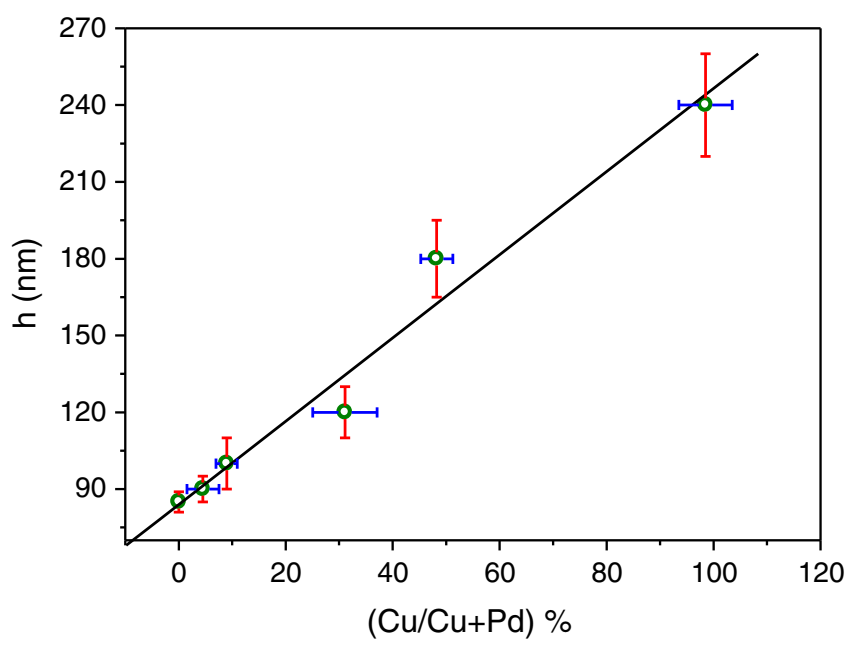

Figure 6. Variation in grating thickness with atom percentage of $\mathrm{Cu}$ deposited on Pd grating.

diffracted from gratings is influenced by the thickness of grating which in turn depends on the amount of $\mathrm{Cu}$ deposition. The changes in DE are quite sharp at lower amounts of $\mathrm{Cu}$ deposition as seen from figure $7 \mathrm{a}$, which illustrates the sensitivity of this technique employed. $D E$ saturates after $30 \%$ of $\mathrm{Cu}$ where $\mathrm{Pd}$ gets fully covered with $\mathrm{Cu}$ and Pd surface is no longer available for deposition. For $\mathrm{Cu}$ deposition above $30 \%$, the DE starts decreasing as the grating structure starts diminishing with excessive growth of $\mathrm{Cu}$ between the gaps. Interestingly, it was found that for $8 \mathrm{~nm}$ increase in thickness following $\mathrm{Cu}$ deposition, the diffraction efficiency increased by $16.4 \%$ from the initial value of $10.2 \%$ (with no $\mathrm{Cu}$ ) (figure $7 \mathrm{~b}$ ). This value is close to the results in the literature where the change in thickness by $\sim 5 \mathrm{~nm}$ due to the CdSe nanocrystals increased the diffraction efficiency by 10-15\% (Shallcross et al 2009).

The above study effectively makes use of the grating action of a CD (Meenakshi et al 2007) but in the form of a Pd grating pattern. Its high resolution enables the use of a simple LED source and photodetector as components of the monitoring device with the illuminated grating area being $\sim 1 \mathrm{~mm}$. This may be contrasted to optical diffraction based monitoring devices using conventional gratings with spacings of few tens of microns where the use of a high resolution detector becomes mandatory as the diffraction angles are considerably smaller. Besides, much larger grating areas are required. With the grating presented in this study, it should be possible to fabricate a diffraction chip with each bit extending over say, 100 microns, yet with little compromise on resolution. 

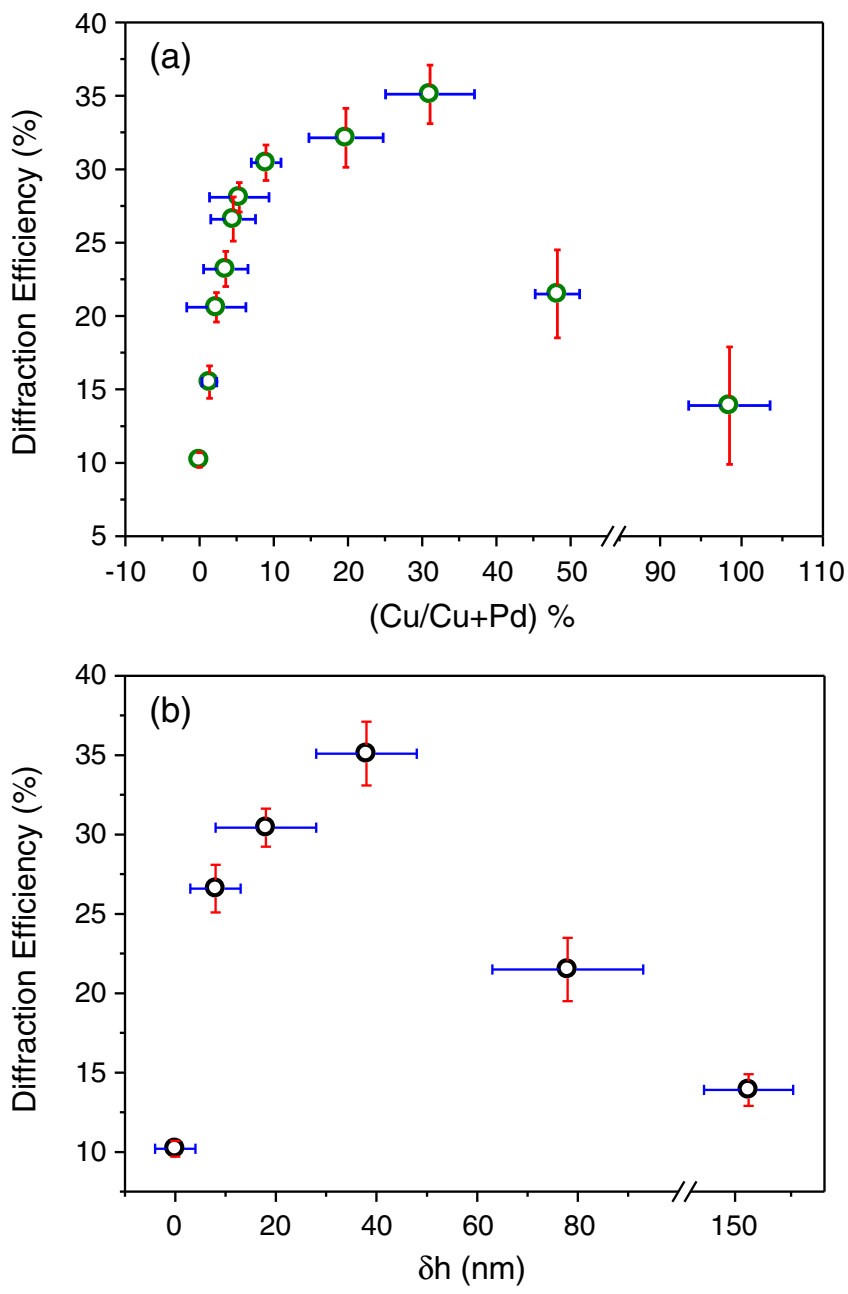

Figure 7. Variation in diffraction efficiency (DE) calculated from measured intensities of diffraction spots with (a) atom percentage of $\mathrm{Cu}$ on $\mathrm{Pd}$ grating and (b) increasing thickness of grating.

\section{Conclusions}

In this study, we have produced Pd diffraction grating structures using a direct micromolding method, employing a PDMS stamp derived from CD as the mold. Pd gratings were obtained on glass substrates by injecting Pd alkylthiolate solution through the mold and metallizing the precursor by subjecting to thermolysis. The obtained structures were essentially replica of the CD track patterns, whose thickness could be adjusted by varying the concentration of thiolate solution. The obtained Pd grating structures were used as optical diffraction devices performing at a high resolution, $\sim 2000$ with a diode laser $(\lambda=650 \mathrm{~nm})$ as the light source. A simple photodiode served as detector in the transmission mode. Using thin Pd grating stripes as seed layer, the electroless $\mathrm{Cu}$ uptake was monitored based on the diffraction efficiency and the results were corroborated with EDS and AFM data. The diffraction grating showed a $16 \%$ increase in the diffraction efficiency for a $8 \mathrm{~nm}$ increment in the grat- ing thickness due to $\mathrm{Cu}$. Thus, the change in grating thickness could be monitored sensitively based on the diffraction measurements. This characterization tool is particularly useful while studying adlayers in the thickness range of few tens of nanometers. Such devices may find applications in circuit fabrication by plating such as ULSI processes, where $\mathrm{Cu}$ deposition on Pd seed catalyst is routinely employed as it will cut down the instrumentation cost, manual time and enable fast production (Ma et al 2009). Future studies will focus on fabrication of diffraction based chips with few tens of lines defining a grating bit.

\section{Acknowledgements}

The authors thank Professor C N R Rao, FRS, for his encouragement. Support from the Department of Science and Technology, Government of India, is gratefully acknowledged. The authors thank Veeco India Nanotechnology Laboratory, JNCASR, for providing AFM facility. (RG) thanks Deepyanti Taneja for initial assistance in organizing the diffraction set up.

\section{Supplementary material}

Supplementary material pertaining to this article is available on the Bulletin of Materials Science website (www.ias.ac.in/ matersci).

\section{References}

Acharya G, Chang C L, Doorneweerd D D, Vlashi E, Henne W A, Hartmann L C, Low P S and Savran C A 2007a J. Am. Chem. Soc. 12915824

Acharya G, Doorneweerd D D, Chang C L, Henne W A, Low P S and Savran C A 2007b J. Am. Chem. Soc. 129732

Acharya G, Chang C L, Holland D P, Thompson D H and Savran C A 2008 Angew. Chem. Int. Ed. 471051

Aithal R K, Yenamandra S, Gunasekaran R A, Coane P and Varahramyan K 2006 Mater. Chem. Phys. 9895

Bailey R C and Hupp J T 2003 Anal. Chem. 752392

Bailey R C, Nam J M, Mirkin C A and Hupp J T 2003 J. Am. Chem. Soc. 12513541

Bhuvana T and Kulkarni G U 2008 ACS Nano 2457

Chen Y, Kung, S C, Taggart D K, Halpern A R, Penner R M and Corn M R 2010 Anal. Chem. 823365

Goh J B, Loo R W, McAloney R A and Goh M C 2002 Anal. Bioanal. Chem. 37454

Goh J B, Loo R W and Goh M C 2005 Sens. Actuators B106 243

Halpern A R, Nishi N, Wen J, Yang F, Xiang C, Penner R M and Corn R M 2009 Anal. Chem. 815585

Hidber P C, Helbig W, Kim E and Whitesides G M 1996 Langmuir 121375

Inberg A, Glickman E, Asher T, Fishelson N and Shacham Y 2009 Surf. Coat. Technol. 204520 
Kane R S, Takayama S, Ostuni E, Ingber D E and Whitesides G M 1999 Biomaterials 202363

Konkola P T, Chen C G, Heilmann R K, Joo C, Montoya J C, Chang C H and Schattenburg M L 2003 J. Vac. Sci. Technol. B21 3097

Ma C, Ye W, Shi X, Chang Y, Chen Y and Wang C 2009 Appl. Surf. Sci. 2553713

Massari A M, Stevenson K J and Hupp J T 2001 J. Electroanal. Chem. $\mathbf{5 0 0} 185$

Meenakshi V, Babayan Y and Odom T W 2007 J. Chem. Edu. 84 1795

Morhard F, Pipper J, Dahint R and Grunze M 2000 Sens. Actuators B70 232

Nakajima F, Hirakawa Y, Kaneta T and Imasaka T 1999 Anal. Chem. 712262
Pan Z W, Mahurin S M, Dai S and Lowndes D H 2005 Nano Lett. 5 723

Radha B and Kulkarni G U 2009 Small 52271

Ryckman J D, Liscidini M, Sipe J E and Weiss S M 2010 Appl. Phys. Lett. 96171103

Sarov Y E, Capek I, Ivanov T B, Ivanova K Z, Sarova V A and Rangelow I W 2007 Nano Lett. 8375

Sendroiu I E and Corn R M 2008 Biointerphases 323

Shallcross R C, Chawla G S, Marikkar F S, Tolbert S, Pyun J and Armstrong N R 2009 ACS Nano 33629

St. John P M, Davis R, Cady N, Czajka J, Batt C A and Craighead H G 1998 Anal. Chem. 701108

Tsay Y G, Lin C I, Lee J, Gustafson E K, Appelqvist R, Magginetti P, Norton R, Teng N and Charlton D 1991 Clin. Chem. 371502 